\title{
INFLUENCE OF THE LAYERED STRUCTURE OF THE IONOSPHERE ON THE NATURAL ELF RADIATION SPECTRA
}

\author{
V.A. Rafalsky \\ Institute of Radio Astronomy, Ukrainian Academy of Sciences \\ Kharkov, 310002, Ukraine
}

\begin{abstract}
This paper reports on some propagation effects occurring while the ionosphere consists of several layers. A few simple model problems were considered to show in which way this multi-layer structure can yield a new set of ELF resonance phenomena. The resonances appear in the space between the Earth and layer interfaces or inside the layers and are liable to give rise to peaks or minima in the frequency dependencies of ELF electromagnetic fields both for the cosmic and Earth sources. Calculations been made for the real ionosphere models and distant Earth's sources showed that such a mechanism can explain some experimental data including the effect of "The Evening Ionosphere Radiation" by Shecotov and Molchanov (Geomagnetism and Aeronomy, 25, N4,598, 1985).
\end{abstract}

1. Introduction

Shecotov and Molchanov (1985) have detected an effect that was a spectral peak of ELF natural electromagnetic noise observed at the auroral latitude. The peak arose in the frequency band of $100-200 \mathrm{~Hz}$ in the evening and then slowly shifted towards the high frequencies. The phenomenon being named "The Evening Ionosphere Radiation" was explained as radiation of a narrow-band source superposed on the regular background noise.

Measurements were performed at Lekhta observatory $\left(60^{\circ} \mathrm{N}\right.$, $112^{\circ}$ E) during the second half of the year of 1983. The horizontal North-South component of $\mathrm{H}$ field was analyzed. The effect have been found in 53 from 65 twenty-four-hour surveys. The spectral peak usually arose after sunset and was persistent during 1-3 hours. The upper frequency of the process was reaching $400 \mathrm{~Hz}$ in some records.

A similar peak can be observed at middle latitudes as follows from experimental data by Bashkuyev et al (1989). During the measurements performed near Baikal lake $\left(53^{\circ} \mathrm{N}\right.$, $108^{\circ}$ E) in March 1988 , a wide spectral peak of the horizontal magnetic field component was observed all night long at the frequency of about $400 \mathrm{~Hz}$.

In the present paper a new explanation of the ELF noise spectra features is introduced.

The Earth's ionosphere consists of several layers. Electron density at the layer boundary is changed more sharply than inside the layer. It follows that there is a principal possibility to observe reflections from $E$ and $F$ ionosphere layers and associated resonance phenomena. 
The eigenfrequencies $f_{p}$ of such resonances can be estimated as follows:

$$
f_{p}=p c / 2 h
$$

where $\mathrm{p}$ is the mode number, $\mathrm{c}$ is teh light velocity, $\mathrm{h}$ is the effective height of the reflection level (taking into account the refractive index of propagation medium).

The frequencies $f_{p}$ fall into the frequency range of some kilohertz when the waves reflect from the lower boundary of ionosphere. We will see hereinafter that if there are any boundaries within the ionosphere, a new succession of resonance frequencies appear down to hundreds or even tens of hertz.

H.Poeverlein (1961) was the first to point out the possibility of the occurrence of the resonance phenomena in the space bounded by the ground and the interface between $E$ and $D$ ionosphere layers. By such a reason the peak in the calculated field spectrum at the frequency of $440 \mathrm{~Hz}$ was explained.

As follows from calculations by Galejs (1972) resonances are feasible to occur in the range of tens hertz as well. Contrary to the Shumann resonances, these ones are transversal by their nature. They result from the standing waves within the lower part of the ionosphere below the $F$ layer and are attended with peaks in the frequency dependencies of the main mode attenuation.

2. Model problem for the eigenfrequencies

We will consider both the eigenfrequency problem of the natural resonator and its excitation in two ways: by the plane wave penetrating through the ionosphere and with a point source placed just above the ground. In all cases the problems are solved within a small size region (in comparison to the Earth's radius) taking the resonator as a flat system. In addition the ground is supposed to be perfectly conducting.

We begin with the two layer isotropic model of the ionosphere (Fig.1). The fields in the lower layers are readily represented as a pair of plane waves propagating along $z$ axis in opposite directions. There will be only one wave in the upper layer - the upgoing one.

Satisfying the boundary conditions we obtain the following dispersion equation (for brevity only TM solutions will be

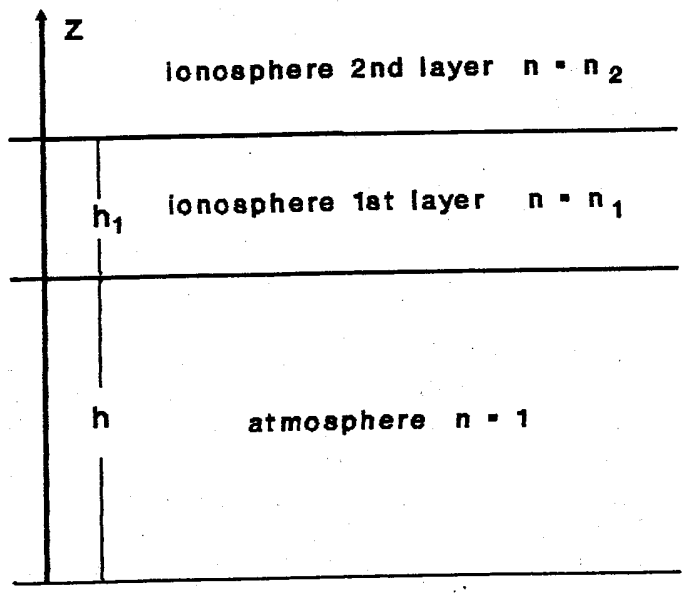

Earth $6=\infty$

Fig. 1. Flat model with two layer ionosphere. considered). 


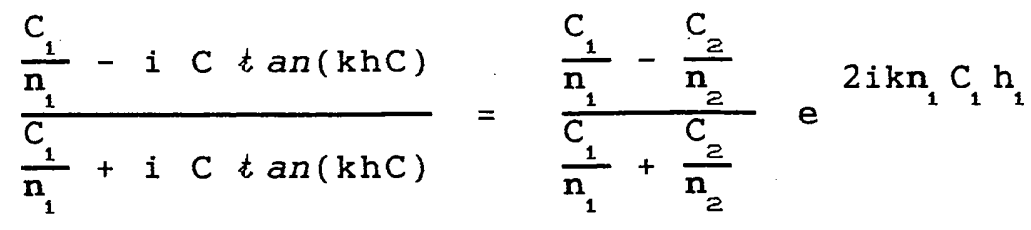

Here $C_{1} C_{1}, C_{2}$ are cosine of the angles between the normal to the boundaries and propagation direction in the media with refractive indices $\mathbf{n}=1, \mathbf{n}_{1}, \mathbf{n}_{2}$ respectively, $c_{i}^{2}=1-\left(1-C^{2}\right) / \mathbf{n}_{i}^{2}$, $(i=1,2) ; \mathrm{k}=\frac{\omega}{\mathrm{c}}, \mathrm{i}=\sqrt{-1}$.

If the angle of incidence (i.e. C value) is preset, the series of the system eigenfrequencies can be obtained - complex ones in general case. And vice versa, if the frequency is fixed, the succession of complex angles will be resulted from Eq. (1). Each of those corresponds to an associated normal wave (mode) of the Earth - ionosphere waveguide.

Results of eigenfrequensies computation from eq. (1) are shown in Fig.2. Parameters of the model of Fig.1 were specified as: $n_{1}=10+\beta i, n_{2}=30, h=h_{1}=60 \mathrm{~km}$, where $\beta$ will be varied, and $C$ as unity (normal incidence). Supposing $n$ is real $(\beta=0)$, the eigenfrequencies being marked by points in the complex plane fall along the real axis. When $\beta$ increases the eigenfrequencies fall along a straight line sloping more steeply. The exception is the root falling near the resonant frequency of the space between the Earth and the lower edge of the ionosphere (in. Fig. 2 - near $2.5 \mathrm{kHz}$ ). First its imaginary part increases and then begins to decrease dropping out of the general linear dependence. This particular wave does not "feel" the boundary within the ionosphere.

Even though the considered qualitative example does not fit well the real properties of the ionosphere we can definitely conclude the following: (i) resonance phenomena may appear in the space between the ground and the ionosphere layer interfaces, (ii) these resonances lie at frequencies lower than those appearing between the ground and the lower edge of the ionosphere.

3. Model problems for the fields

We include two more model problems just to see in which way the resonances will affect observable fields.

Consider the penetration of a plane wave into the space between the ground and two-layer ionosphere (Fig.1). We will omit the unwieldy equations confining ourselves to calculation of the spectra of the horizontal magnetic field component (Fig.3). It is obvious that the resonance peak in Fig.3 appears because of a standing wave in the space between the ground and the ionosphere layer interface. This peak declines with the increase of losses within the ionosphere $\left(\operatorname{Im} \mathbf{n}_{1}\right)$. 


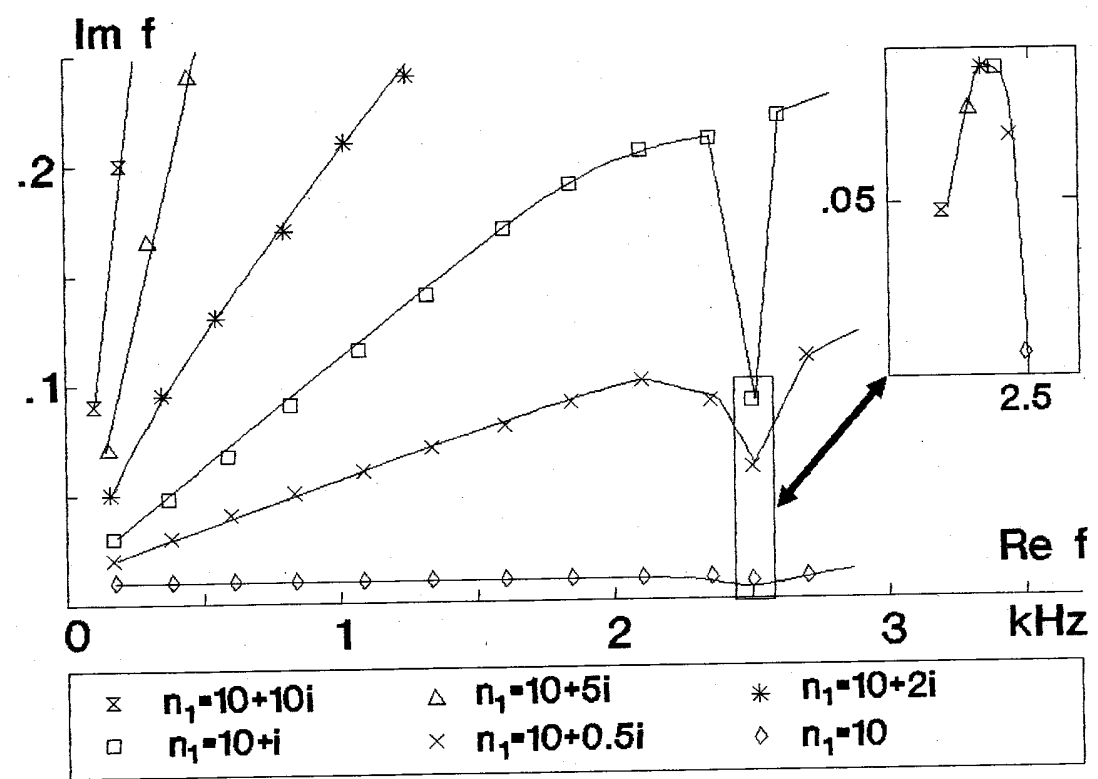

Fig. 2. Eigenfrequencies for the model of Fig. 1.

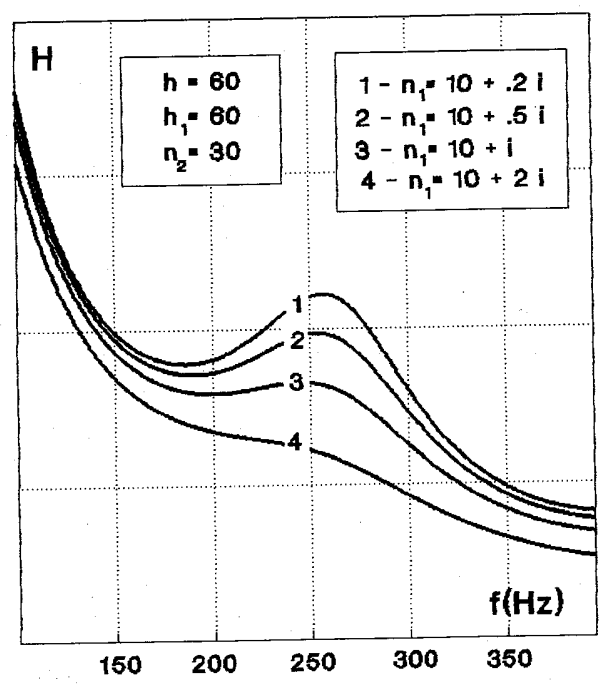

Fig. 3. Horizontal magnetic field excited with a plane wave (model of Fig. 1).

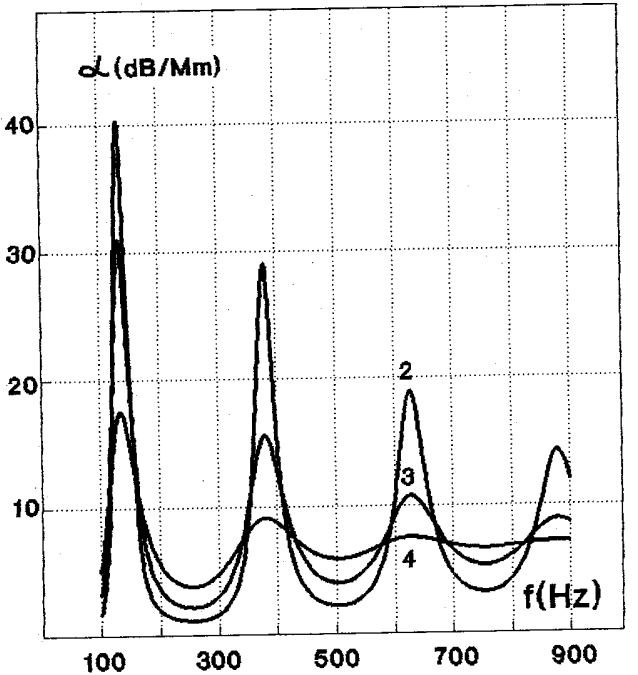

Fig. 4. Attenuation of the main mode (model of Fig. 1). 
Similar field dependencies can also be observed when the system of two-layer ionosphere is excited with a point source and the fields are measured somewhere on the ground, even though the problem formulation is quite different. Fields in such a waveguide are usually represented as a sum of normal waves (modes). Taking into account the previous estimations we shell restrict our consideration to the frequencies below one kilohertz where only one mode propagates having the attenuation $\alpha(\mathrm{dB} / \mathrm{Mm})=0.182 \mathrm{f}$ Im $\mathrm{S}_{0}$. When $\mathrm{n}_{2} \equiv \alpha$, then $\left|\mathrm{n}_{1}\right|>>1$ the dispersion equation (1) can be transformed to the form

$$
\eta \tan \eta=-i k h \delta \quad, \quad(2)
$$

where

$$
\begin{aligned}
& \eta=k h C, \quad \delta=-\frac{i}{h} \tan \left(k n_{1} h_{1} c_{1}\right), \\
& c=\sqrt{1-s^{2}}, \quad c_{1}=\sqrt{1-s^{2} / n_{1}^{2}} .
\end{aligned}
$$

Expression (2) coincides in form with the dispersion equation for the planar impedance waveguide, but differs in that impedance $\delta$ is a strongly dispersing value. When $n_{1}$ is real, $\delta$ becomes a periodical function of frequency. The periodicity of $\delta$ is related directly to the standing waves in the ionosphere layer with $n_{1}$. When $n_{1}$ becomes complex value those oscillations acquire damping that leads to oscillating dependence of the main mode attenuation. Such curves obtained from numerical solution of Eq. (1) are seen in Fig. 4 . If Im $\mathbf{n}_{1}$ is high enough the only attenuation peak - resonance absorption, and a weak minimum - resonance reflection may be observed.

4. Fields for the real ionosphere models

Let us consider the same problem for the ionosphere models whose parameters such as electron concentration and effective collision frequency are the smooth functions of the height $z$. The Earth's vertical magnetic field is introduced as 0.3 Oe. Four profiles of the electron concentration $\mathrm{N}_{\mathrm{e}}(\mathrm{z})$ (Fig. 5) contain more steep gradient at a height about $100 \mathrm{~km}$ modeling the upper boundary of $D$ layer. Such a sequence of $\mathrm{N}_{e}$ profiles may appear while the ionosphere switches from day to night conditions. The technique of recalculating of the matrix admittance and then numerical solution of the dispersion equation for the anisotropic Earth - ionosphere waveguide was used (See Budden, 1961, Galyuk and Ivanov, 1978).

In Fig. 6 results of calculation of the main mode attenuation are shown. There are four frequency dependencies corresponding to the models of Fig. 5. Each of these curves contains a peak of attenuation shifting in frequency from one 
model to another, i.e. from day to night conditions. Using the conception that have been developed above we can conclude the peak to be affected by resonance absorption of electromagnetic waves within D ionosphere layer.

The maximum of attenuation leads to a field spectrum minimum when the waveguide is excited with distant wideband sources such as lightning discharges. If parameters of the ionosphere do change, the position of the peak versus the frequency may shift. That is shown in Fig. 7 where horizontal magnetic field spectra for the models of Fig. 5 are depicted. The source is supposed to be a vertical electric dipole placed 5000 $\mathrm{km}$ away. When changing over from one model to the next the field minimum and an attached maximum move towards high frequencies.

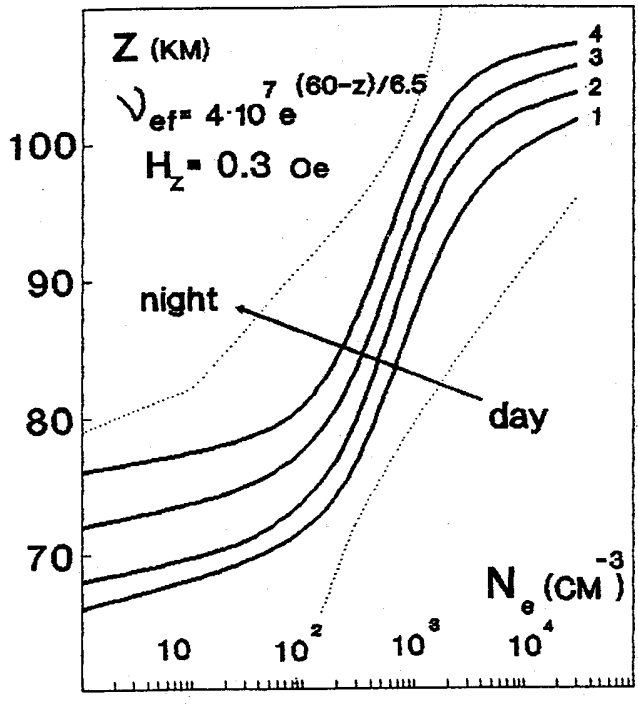

Fig. 5. Ionosphere models with smooth dependence of parameters of height.

\section{Discussion and conclusions}

The resonance mechanism considered above allows explaining behavior of electromagnetic noises in the frequency range of $100-500 \mathrm{~Hz}$. Moreover, in two different cases when excited both with a distant cosmic source and of a terrestrial origin, similar results may appear. Field spectra have local minimum and maximum coupled together. Their position in the frequency axis is determined by "optical thickness" of the resonant space. This is the space between the ground and D-E layer interface in the first case and within $D$ layer in the second case.

The extremes can stay put for a long time as in Bashkuyev et al. (1989) or move in frequency just as in shecotov and Molchanov (1985) if the electron concentration profile or other ionosphere parameters change.

On this basis one can formulate and solve inverse problems, e.g. to determine effective (optical) thickness of the ionosphere layer below the reflection level from the measured frequencies of the extremes. The curves become less pronounced with the growth of energy losses within that layer.

It is noticeable that the frequency drift just as in the Radiation of the Evening Ionosphere effect is observed almost only during the evening hours, whereas the similar ionosphere transformations (ignoring direction of the changes) occur either in the evening or in the morning. We can adduce two 


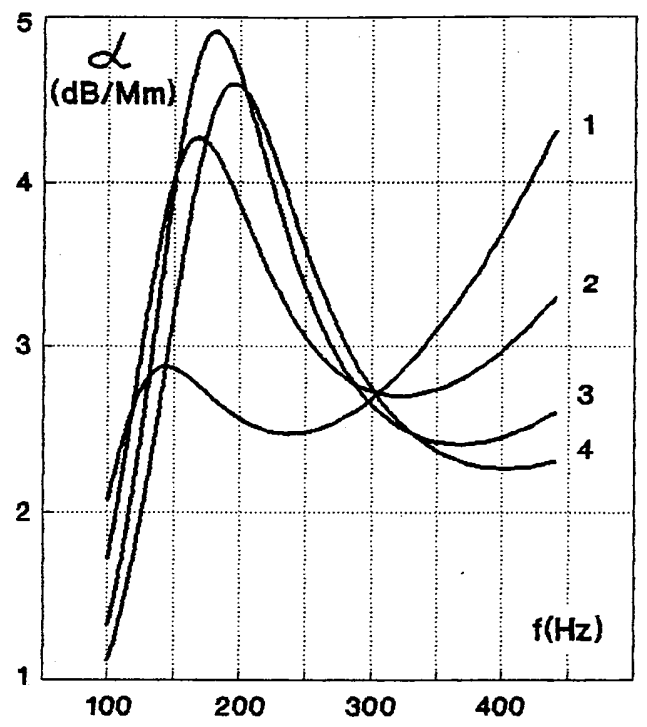

Fig. 6. Attenuation of the main mode (models of Fig.5).

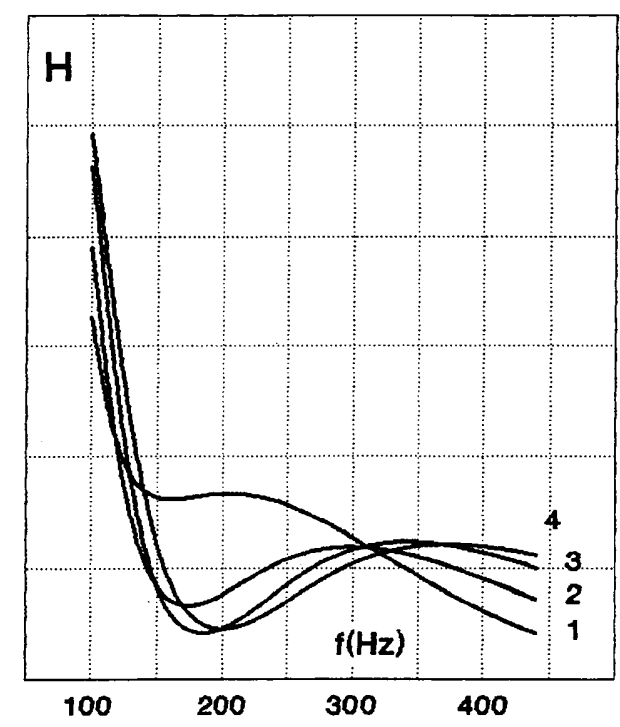

Fig. 7. Horizontal magnetic field excited with a point source (models of Fig.5).

possible explanation of this fact. The first is that the morning and the evening sequences of the electron density profiles do not coincide, and the morning absorption in the transition layer may be greater than evening one. The second possible explanation is based on the time - spatial distribution of the lightnings, since the effect is enhanced when propagating along the day - night interface.

Acknowledgement. The author would like to thank A.P.Nickolaenko for his support and useful suggestions.

\section{References}

Bashkuev, Yu.B., V.B.Haptanov, D.G.Buyanova, and E.M.Mitkinov, Spectral features and spatial structure of ELF noise in middle latitudes of Asia., Proc.Int.Symp. on EMC, Nagoya, Vol. 1, P. 44-49, 1989.

Budden, K.G., Radio waves in the ionosphere. Cambridge Univ. Press, 1961 .

Galejs, J. Terrestrial propagation of long electromagnetic waves. Pergamon Press, Oxford, pp.362, 1972.

Galyuk, Yu.P., and V.I.Ivanov, Calculation of characteristics of VLF propagation in the Earth - anisotropic inhomogeneous ionosphere waveguide. Problems of diffraction and radiowave propagation. Vol. 16, Leningrad, 148-154, 1978. 
Poeverlein, H., Resonance of the space between earth and iono sphere. J. Res. NBS-D. 65-D, N5. 465-473, 1961.

Rafalsky, V.A., Resonance phenomena in the transverse crosssection of the Earth - ionosphere space and their influence on the radiowave excitation and propagation. Ph. D. dissertation. Kharkov. Inst. of Radio Astronomy, 1991, (In Russian).

Shecotov, A.Yu., and O.A.Molchanov, ELF radiation of the evening ionosphere on the Earths surface in subauroral latitudes. Geomagnetism and Aeronomy, 25, N4, 598, 1985, (in Russian). 